\title{
Recommendations from the EGAPP Working Group: does genomic profiling to assess type 2 diabetes risk improve health outcomes?
}

\author{
Evaluation of Genomic Applications in Practice and Prevention (EGAPP) Working Group*
}

\begin{abstract}
This recommendation statement is a product of the independent Evaluation of Genomic Applications in Practice and Prevention (EGAPP) Working Group. Although the Centers for Disease Control and Prevention (CDC) provides support to the EGAPP Working Group, including staff support in the preparation of this document, recommendations made by the EGAPP Working Group should not be construed as official positions of the CDC or the
\end{abstract} US Department of Health and Human Services.

\begin{abstract}
Summary of recommendations: The Evaluation of Genomic Applications in Practice and Prevention (EGAPP) Working Group (EWG) found insufficient evidence to recommend testing for predictive variants in 28 variants (listed in Table 1 ) to assess risk for type 2 diabetes in the general population, on the basis of studies in populations of northern European descent. The EWG found that the magnitude of net health benefit from the use of any of these tests alone or in combination is close to zero. The EWG discourages clinical use unless further evidence supports improved clinical outcomes.
\end{abstract}

The EWG found insufficient evidence to recommend testing for the TCF7L2 gene to assess risk for type 2 diabetes in high-risk individuals. The EWG found that the magnitude of net health benefit from the use of this test is close to zero. The EWG discourages clinical use unless further evidence supports improved clinical outcomes.

On the basis of the available evidence for both the scenarios, the overall certainty of net health benefit is deemed "low."

Rationale: It has been suggested that genomic profiling in the general population or in high-risk populations for type 2 diabetes might lead to management changes (e.g., earlier initiation or higher rates of medical interventions, or targeted recommendations for behavioral change) that improve type 2 diabetes outcomes or prevent type 2 diabetes. The EWG found no direct evidence to support this possibility; therefore, this review sought indirect evidence aimed at documenting the extent to which genomic profiling alters type 2 diabetes risk estimation, alone and in combination with traditional risk factors, and the extent to which risk classification improves health outcomes.

Analytic validity: Assay-related evidence on available genomic profiling tests was deemed inadequate. However, on the basis of existing technologies that have been or may be used, the analytic sensitivity and specificity of tests for individual gene variants might be at least satisfactory.

Clinical validity: Twenty-eight candidate markers were evaluated in the general population. Evidence on clinical validity was rated inadequate for 24 of these associations (86\%) and adequate for 4 (14\%).
Inadequate grades were based on limited evidence, poor replication, existence of possible biases, or combinations of these factors. Type 2 diabetes genomic profiling provided areas under the receiver operator characteristics curve of $55 \%-57 \%$, with 4,8 , and 28 genes. Only TCF7L2 had convincing evidence of an association with type 2 diabetes with an odds ratio of 1.39 (95\% confidence interval: 1.33-1.46).

TCF7L2 was evaluated for high-risk populations, and the overall odds ratio was 1.66 (95\% confidence interval: $1.22-2.27)$ for association with progression to type 2 diabetes.

Clinical utility: No studies were available to provide direct evidence on the balance of benefits and harms for genetic profiling for type 2 diabetes alone or in addition to traditional risk factors in the general population.

Evidence for high-risk populations and TCF7L2 was inadequate on the basis of two identified studies. These studies found close to zero additional benefit with the addition of genomic markers to traditional risk factors (diet, body mass index, and glucose tolerance).

Contextual issues: Prevention of type 2 diabetes is a public health priority. Improvements in the outcomes associated with genomic profiling could have important impacts. Traditional risk factors (e.g., body mass index, weight, fat mass, and exercise) have an advantage in clinical screening and risk assessment strategies because they measure the actual targets for therapy (e.g., fasting plasma glucose and medical interventions). To be useful in predicting disease risk, genomic testing should improve the predictive value of these traditional risk factors. Some issues important for clinical utility remain unknown, such as the level of risk that changes intervention, whether long-term disease outcomes will improve, how individuals being tested will understand/respond to test results and interact with the health-care system, and whether testing will motivate behavior change or amplify potential harms.

Genet Med 2013:15(8):612-617

Key Words: EGAPP; genetic testing; recommendation; risk; type 2 diabetes

${ }^{\star}$ EGAPP Working Group: Chair: Ned Calonge, MD, MPH (Colorado Trust); Members: Jonathan S. Berg, MD, PhD (Department of Genetics, UNC School of Medicine); Doug Campos-Outcalt, MD, MPA (Department of Family and Community Medicine, University of Arizona College of Medicine); Benjamin Djulbegovic, MD, PhD (Co-Director, Clinical \& Translational Science Institute, and Director, Center for Evidence Based Medicine and Research, University of South Florida); Theodore G. Ganiats, MD (Department of Family and Preventive Medicine, University of California, San Diego); Cecile Janssens, PhD (Rollins School of Public Health, Emory University); Roger D. Klein, MD, JD (Department of Molecular Pathology Cleveland Clinic Foundation); Kenneth Offit, MD, MPH (Clinical Genetics Service, Memorial Sloan-Kettering Cancer Center); Stephen G. Pauker, MD, MACP, FACC, ABMH (Division of Clinical Decision Making, Informatics and Telemedicine, Department of Medicine, Tufts Medical Center); Margaret Piper, PhD, MPH (Blue Cross/Blue Shield Association Technology Evaluation Center); Carolyn Sue Richards, PhD, FACMG (Oregon Health \& Science University); Ora L. Strickland, PhD, DSc (Hon.), RN, FAAN (Florida International University); Sean Tunis MD, MSc (Center for Medical Technology Policy); Marc S. Williams, MD (Genomic Medicine Institute, Geisinger Health System); and Doris Zallen, PhD (Department of Science and Technology in Society, Virginia Tech). Correspondence: Michael P. Douglas (mrd4@cdc.gov) 
Table 1 Summary of gene/marker-disease associations for type 2 diabetes in Caucasians

\begin{tabular}{|c|c|c|c|c|c|c|}
\hline $\begin{array}{l}\text { Genes included on test } \\
\text { panels for type } 2 \text { diabetes }\end{array}$ & deCODE T2 & deCODEme & 23andMe & Navigenics & Baylor panel & Variant \\
\hline TCF7L2 & $\bullet$ & $\bullet$ & $\bullet$ & $\bullet$ & $\bullet$ & rs7903146 \\
\hline CDKAL1 & $\bullet$ & $\bullet$ & $\bullet$ & $\bullet$ & $\bullet$ & rs7756992 \\
\hline CDKN2A/B & $\bullet$ & $\bullet$ & $\bullet$ & & & rs10811661 \\
\hline PPARG & $\bullet$ & $\bullet$ & $\bullet$ & $\bullet$ & $\bullet$ & rs1801282 \\
\hline HHEX & & $\bullet$ & $\bullet$ & $\bullet$ & $\bullet$ & rs1111875 \\
\hline SLC3OAB & & $\bullet$ & $\bullet$ & $\bullet$ & $\bullet$ & rs13266634 \\
\hline KCNJ11 & & • & $\bullet$ & $\bullet$ & $\bullet$ & rs5219 \\
\hline WSF1 & & $\bullet$ & $\bullet$ & $\bullet$ & & rs10010131 \\
\hline IGF2BP2 & & $\bullet$ & $\bullet$ & $\bullet$ & & \\
\hline BCL11A & & $\bullet$ & & & & \\
\hline THADA & & $\bullet$ & & & & \\
\hline NOTCH2 & & $\bullet$ & & $\bullet$ & & \\
\hline FTO & & & & $\bullet$ & & \\
\hline JAZF1 & & $\bullet$ & & $\bullet$ & & \\
\hline TCF2 & & $\bullet$ & & $\bullet$ & & \\
\hline TSPAN8 & & & & $\bullet$ & & \\
\hline MTNR18 & & $\bullet$ & & & & \\
\hline ADAMTS9 & & & & $\bullet$ & & \\
\hline KCNQ1 & & $\bullet$ & & $\bullet$ & & \\
\hline GCK & & $\bullet$ & & & & \\
\hline PROX1 & & • & & & & \\
\hline GCKR & & $\bullet$ & & & & \\
\hline$D G K B$ (7p21.1) & & • & & & & \\
\hline TSPANG & & $\bullet$ & & & & \\
\hline loc441171 & & & & $\bullet$ & & \\
\hline LGR6 & & $\bullet$ & & & & \\
\hline Chr11.41871942 & & & & $\bullet$ & & \\
\hline $12 q 13$ & & & & & $\bullet$ & \\
\hline
\end{tabular}

\section{CLINICAL CONSIDERATIONS}

\section{Definitions used by the EGAPP}

- Analytic validity refers to a test's ability to accurately and reliably measure the genotype or analyte of interest.

- Clinical validity defines the ability of the test to accurately and reliably identify or predict the intermediate or final outcomes of interest. This is usually reported as clinical sensitivity and specificity.

- Clinical utility defines the balance of benefits and harms associated with using the test in practice, including improvement in measureable clinical outcomes and added value in clinical management and decision making as compared with not using the test.

- Credibility refers to the likelihood that an association exists after some evidence has been accumulated.

\section{Patient population under consideration}

These recommendations apply to the general population and high-risk population (those with impaired fasting glucose) of adults without known preexisting type 2 diabetes (T2D), regardless of family history and other typical risk factors.

\section{Considerations for practice}

These tests have become available through primary-care clinician offices as well as through direct-to-consumer marketing. Patients may ask about such tests or bring results of completed tests to their physicians for advice or consultation. Physicians should routinely consider well-established recommendations for T2D risk assessment in the primary-care setting (e.g., those regarding impaired fasting glucose, body mass index, weight, and fat mass). In addition, all patients should be consistently counseled regarding appropriate physical activity and nutrition behaviors to reduce T2D risk. On the basis of the available evidence, it is unclear how the results of genomic profiling should modify patient care to improve outcomes.

\section{BACKGROUND AND CLINICAL CONTEXT FOR THE RECOMMENDATION}

T2D is a major contributor to morbidity and mortality in the United States. Diabetes mellitus refers to a group of metabolic diseases that are characterized by chronic elevations in plasma glucose. Up to $95 \%$ of all diabetes is considered type 2 (T2D), which is also known as "adult-onset" or "non-insulin dependent 
diabetes." The metabolic mechanisms for T2D include insulin resistance, impaired insulin secretion, and increased hepatic glucose production. Complications include neuropathy, retinopathy, periodontal disease, and accelerated development of cardiovascular disease. Approximately 1.9 million people aged 20 years or older were newly diagnosed with diabetes in 2010 in the United States. ${ }^{1}$ T2D is becoming more common in the United States and throughout many regions of the world. The T2D prevalence in youths aged 10 years and older is 8.5 per 100,000 people in this age group, whereas the prevalence is 0.4 per 100,000 in youths $<10$ years of age. ${ }^{1}$ The prevalence is even higher among adults aged 65 years and older, with 10.9 million, or $26.9 \%$, of all people in this age group being affected. ${ }^{1}$ In addition, the prevalence of $\mathrm{T} 2 \mathrm{D}$ varies by race/ethnicity, with higher rates among African Americans (11.4\%), Hispanic/Latino Americans (8.2\%), Native Americans (8.2\% among Alaska Natives and 27.8\% among certain Native American tribes from the Southwest), and some Asian American and Pacific Islander groups. Furthermore, the Centers for Disease Control and Prevention estimates as many as $27 \%$ of individuals with T2D are undiagnosed, and factors such as an aging population and increasing rates of obesity are expected to further increase the prevalence.

A family history of diabetes is a major risk factor for the disease and is often included in a variety of tools designed to detect either people at risk of diabetes or people with undiagnosed diabetes. One of the reasons to screen for diabetes is that it has a prolonged asymptomatic phase, and early treatment leads to better outcomes. As a result, researchers have developed a variety of simple tools to identify high-risk individuals for diabetes in populations. ${ }^{2}$ It has been amply documented that having one or more first-degree relatives with T2D increases the odds of having the disease compared with someone without such relatives. The estimations vary, but the odds usually range from two to six times more likely. ${ }^{3}$ Moreover, a long-term study reported that the cumulative prevalence of T2D at 80 years of age is $\sim 3.5$ times higher (38 vs. 11\%) for people with a first-degree relative with T2D as compared with people without any affected relative. ${ }^{4}$

Personalized medicine using genomic techniques has shown great promise for diagnosis, prognosis, prediction of treatment response (pharmacogenomics) in pharmacogenomic medicine, treatment, and risk assessment. Risk assessment using molecular markers (e.g., single-nucleotide polymorphism (SNP) panels) can be used to estimate a person's risk for developing some diseases and may allow for interventions in high-risk populations. Several commercial and noncommercial entities have identified SNPs that are associated with the risk of developing $\mathrm{T} 2 \mathrm{D}$ and are now offering diabetes risk testing individually, or as a part of a panel involving risk profiling for chronic diseases, in either the general populations or the high-risk populations.

The EWG was established in 2005 to support the development of a systematic process for assessing the available evidence for rapidly emerging genetic tests for clinical practice. This independent, multidisciplinary panel selected this topic to commission an evidence report to review the validity and utility and highlight critical knowledge gaps, in order to provide guidance on appropriate use of the available genetic tests in specific clinical scenarios using the methodology outlined by the EWG in its methodology publications. ${ }^{5,6}$ The analytic validity (technical test performance), clinical validity (the strength of association that determines the test's ability to accurately and reliably identify or predict the disorder of interest), and clinical utility (balance of benefits and harms when the test is used to influence patient management) are systematically reviewed. ${ }^{5}$ The evidence review ${ }^{7}$ synthesizes the available evidence to inform this EGAPP recommendation in an effort to provide guidance on the use of SNP panels for risk assessment of T2D. Given that randomized trials have demonstrated that changing lifestyle factors (e.g., weight loss, improved diet, and increased physical activity) can result in decreased risk for diabetes, the possible knowledge of an individual's T2D increased risk by genetic testing could improve overall health outcomes. ${ }^{8-15}$

\section{Descriptions of tests and intended use claims}

Four companies offering five genomic tests for T2D were identified in July 2010, and any update to the panels was verified in July 2012. The test panels included between 1 and 28 genes (Table 1) on one or more panels. Multigene/marker test panels were offered from several companies (e.g., deCODE, Navigenics), most often with the intention of predicting risk of future disease in patients from the general population. deCODE offers the deCODE T2 test with two components: risk prediction in the general population using four markers and risk of conversion of prediabetes to diabetes in a high-risk population using the TCF7L2 marker (http://www.decodehealth.com/documents/ cms/sample_reports/deCODET2DSampleReport.pdf). This recommendation is restricted to tests with these specific indications. On the basis of the clinical scenarios presented by the companies offering these test panels, the EWG determined it would be appropriate to commission an evidence-based review to address the questions regarding the same specific clinical scenarios:

- Does the use of a multigene panel to estimate lifetime risk of developing T2D improve patient outcomes?

- Does the use of TCF7L2 testing in a high-risk population to determine short-term (3-4 years) risk of developing T2D improve outcomes?

This EGAPP recommendation statement provides a brief summary of the supporting scientific evidence from the commissioned evidence review ${ }^{7}$ used by the EWG to make recommendations regarding the use of genomic profiling in the general population of adults and in a high-risk population of adults.

\section{REVIEW OF SCIENTIFIC EVIDENCE}

\section{Methods}

The EGAPP Initiative was developed by the Office of Public Health Genomics at the Centers for Disease Control and Prevention to support a rigorous, evidence-based process for evaluating genetic tests and other genomic applications that are in transition from research to clinical and public health 
practice in the United States. ${ }^{5}$ The EWG-commissioned evidence review was contracted by the National Office of Public Health Genomics and performed by a collaboration of external consultants and four EGAPP staff members. A technical expert panel that included three EWG members and an additional consultant provided expert guidance during the course of the review. The final EWG recommendation statement was formulated on the basis of magnitude of effect, certainty of evidence, and consideration of contextual factors. ${ }^{5}$ Credibility of evidence is based on the Venice criteria, which were specifically designed to evaluate gene/marker disease associations. Credibility is graded as being "strong," "moderate," or "weak," on the basis of the amount of evidence, replication, and protection from bias. ${ }^{16}$

\section{Technology description}

In general, genotyping methods have involved discrimination of alleles by primer extension, hybridization, ligation or enzymatic cleavage, and detection using fluorescence, mass, gel electrophoresis, or chemiluminescence. Mistaken alleles, allelic dropout (i.e., amplification of only one of two alleles in a heterozygous individual), and other genotyping errors can result from a number of causes. These have included interaction with flanking DNA sequences, low quality/quantity of the DNA in samples, laboratory problems related to reagents/protocols/ equipment, and human error (e.g., sample mislabeling or contamination, data entry, and interpretation mistakes). Less is known about causes of genotyping errors in newer technologies (e.g., multiplex assays, chips, and SNP arrays) used in routine clinical practice and their potential impact on patient results.

\section{Analytic validity}

For the review, analytic validity can be defined in terms of the identification of a specific gene variant. Five genomic panels were identified that included 28 different markers. The following conclusions concerning analytic validity were made:

- In some instances, insufficient information was provided to identify the specific variant tested within a specific gene. In addition, no published literature was found on the testing platforms used by the laboratories offering the test.

- External proficiency testing was not available for the 28 markers included on these genomic panels.

- deCODE Genetics laboratory (Reykjavik, Iceland), one of the companies offering two of the genomic panels, had previously provided in-house data on the analytic methodology used, as well as estimates of analytic performance for its CardioGenomic Health Panel. ${ }^{17}$ It is assumed the same platform and technology are being used in the T2D genomic panel.

- deCODE Genetics laboratory is Clinical Laboratory Improvement Amendments licensed and College of American Pathologists accredited. Representatives provided information about the platform and methodology used (including a methodology publication), test results compared with bidirectional sequencing, and replication (short term and long term), and reported using blinded samples for internal quality assurance.

Analytic validity conclusions. In-house data for analytic validity previously received from deCODE on a similar test was encouraging but was graded as level 4 evidence. There is inadequate evidence that the genomic profiling tests identified in this report have analytic validity, but platforms exist that could allow at least satisfactory sensitivity and specificity.

\section{Clinical validity}

In this context, clinical validity, expressed as odds ratios (ORs), assesses how effectively the at-risk variants of the genes/markers predict T2D risk in general and high-risk populations. In addition to individual ORs for each marker/disease association, a "best-case scenario" model of the combination of 4,8 , and 28 markers with the strongest evidence was created for the general population. Findings include:

- The quality of evidence for clinical validity varies widely among the 30 markers.

- The most credible evidence of a gene/marker-disease association is for the TCF7L2 marker and T2D. This association is highly reproducible, is unlikely to be influenced by major biases, and has the largest effect size documented for any gene with at least strong credibility $(\mathrm{OR}=1.39)$ in the general population. However, the credibility of evidence is poor in the high-risk population.

- Credible evidence of a gene-disease association for $C D K N 2 A / B$ and T2D is available. The association is highly reproducible, is unlikely to be influenced by major biases, and has the largest effect size documented for any gene with at least strong credibility $(\mathrm{OR}=1.22)$ in the general population.

- Six other markers were formally evaluated and have at least some credible evidence (moderate to weak).

- Several markers have associations based on only a few small, heterogeneous studies. These effect sizes are suspect due to important possible biases.

- The cumulative effect (cOR), when displayed as a receiveroperator characteristic curve, produced an area under the curve of $54.7 \%$ for the four strongest credible genes associated with T2D. The cOR, when displayed as a receiveroperator characteristic curve, produced an area under the curve of $55.1 \%$ for the eight strongest credible genes associated with T2D and an area under the curve of $57.0 \%$ for all the markers associated with T2D in the tests evaluated (28 markers). An area under the curve of $100 \%$ is a perfect diagnostic test, whereas a value of $50 \%$ indicates the test provides no useful information. Using a $\mathrm{COR}$ of 1.38 as a cutoff (the demarcation between the 12th and 13th of the 20 intervals), $7.2 \%$ of individuals with $\mathrm{T} 2 \mathrm{D}$ and $4.5 \%$ of normal individuals received cORs at or above this level. This interval was chosen in order to ensure a false-positive rate of $5-10 \%$. On the basis of these performance 
characteristics, the combination of all genomic markers would not be considered a useful stand-alone test for T2D risk stratification in the general population.

Clinical validity conclusions. There is convincing evidence that several genes are associated with T2D and that the improvement in prediction when added to traditional risk factors is close to zero. There were eight genes evaluated and two of these (25\%) had strong credibility (TCF7L2 and $C D K N 2 A / B)$. Among the remaining combinations, two (25\%) are considered to have moderate credibility and four (50\%) weak credibility. Without considering the four combinations with weak credibility, the ORs range from a high of 1.39 to a low of 0.86 . The combination of all genomic markers would not be considered a useful stand-alone test for T2D risk stratification in the general population.

\section{Clinical utility}

In the setting of adults without known T2D or at high risk for T2D, clinical utility assesses the benefits and harms associated with using genomic profiling tests to estimate risk and guide management as ways of improving health-related outcomes. Benefits might include successfully motivating behavior changes and more appropriately treating patients for whom T2D has been determined to be high risk. Harms might include false reassurance triggering negative or no behavior change in those classified as being at low risk, as well as unnecessary drug therapy for patients incorrectly classified as being at high risk. No direct studies in the available literature assess the clinical utility of T2D genomic profiles in the general population. In an effort to provide some indirect evidence of clinical utility, several studies were identified. In summary:

- One study in general populations of TCF7L2 genotyping alone reported a protective effect of whole-grain intake on diabetes risk exclusively applied to CC (nonrisk) genotype carriers. However, study subjects carrying the T allele (risk) seemed to exhibit no benefit from whole-grain consumption, indicating individuals identified as at risk using TCF7L2 genotyping alone may not benefit from a diet modification.

- A systematic review found evidence that classification of high risk for developing T2D using traditional nongenomic methods or being diagnosed with T2D and medical treatment and/or modifying behavior (e.g., drug treatment, diet, and exercise) may translate into modest benefits without clinical harms. However, the need for quality evidence on direct genomic profiling and benefits to those with classification of high risk for developing T2D or being diagnosed with T2D, and for replication of the results in different clinical settings, was emphasized.

- Two studies assessing the clinical utility of genomic testing showed that knowledge of a genomic test result was associated with short-term positive behavior changes (e.g., adherence to risk-reducing behaviors) in two settings (e.g., familial diabetes and smoking cessation programs). ${ }^{18,19}$
Clinical utility conclusions. There is inadequate evidence of clinical utility for genomic profiles directly with T2D risk in the general or high-risk populations.

\section{Clinical studies}

Genetic testing for risk assessment in the general and the highrisk populations is an active and ongoing area of study. The pending results of four clinical studies could affect this and future recommendations regarding T2D and genomic profiling. The following is from clinicaltrials.gov:

- Molecular and clinical profile of diabetes mellitus and its complications: The study will identify and characterize genetic variants associated with $\mathrm{T} 2 \mathrm{D}$, its risk factors, and its complications (NCT01105858).

- Genetic counseling and lifestyle change for diabetes prevention: The study will examine the impact of diabetes genetic counseling on patient motivation and diseaseprevention behaviors among subjects with prediabetes. Intervention subjects will be provided with their individual diabetes genotype risk score derived from aggregating the combined results of 37 diabetes-riskassociated genetic loci. Controls will not be tested (NCT01034319).

- Genetic testing for type 2 diabetes: This is a 6-month randomized, controlled trial that will evaluate the impact of genetic testing for T2D on psychological, health behavior, and clinical outcomes. Results from this study will inform whether genetic counseling can be effective for communicating disease risk, motivating behavior change, and, ultimately, preventing a complex, chronic disease (T2D) (NCT01060540).

- Effect of type 2 diabetes genetic risk information on health behaviors and outcomes: The study is to assess the clinical utility of a genetic test for T2D risk in combination with standardized risk assessment compared with standardized risk assessment alone and to measure whether changes in perceived risk following genetic testing for T2D risk are correlated with behavior change and increased concern about risk for T2D (NCT00849563).

\section{Contextual issues important to the recommendation}

- T2D is an important public health problem, and improvements in outcomes associated with genomic testing could have important far-reaching impacts.

- The traditional modifiable T2D risk factors have an advantage in clinical screening and risk assessment strategies because they measure the actual targets for therapy (e.g., glucose levels, lipid levels, blood pressure, and body mass index).

- To be useful, genomic testing should provide demonstrable improvement upon the predictive value of traditional risk factors.

- The genetic mechanism of most candidate markers/gene variants is unknown. 


\section{Cost effectiveness}

This review did not include any economic analyses.

\section{Research gaps}

The EWG found the research literature insufficient with important gaps in knowledge, including:

- Little or no available information on the analytic validity of genomic panels, either in the published literature or on the company websites. Often, it was not possible to even determine the testing platform or assay methodology being used.

- The specific markers or gene variants that were included in some of the genomic panels were not found in any published literature.

- Which of the gene/variant associations identified might benefit from further validation and/or analysis in order to improve their credibility?

- How information gained from GWA studies might be helpful in determining the effect size and credibility of existing gene/disease associations?

- Which, if any, of the gene/disease associations identified with moderate or weak credibility might be overestimated due to potential biases (e.g., publication bias)?

- How multiple genomic markers for T2D should be combined, and the types of data needed to inform these models.

- How does the addition of genomic markers for T2D with existing family history add to risk prediction; and in the case of a lack of family history (i.e., adoption cases), do genomic markers provide any risk prediction?

- What methodology should be used to determine the extent to which genomic (or nongenomic) markers add useful information to an existing risk model?

- Alternative strategies for prevention of T2D and how genomic markers might impact these strategies.

- Are there behavioral changes related to providing the results of genomic testing, would these changes plausibly lead to improved health, and what factors might influence these changes (e.g., setting, method of delivery, and change in risk)?

- More research is needed in minority/nonwhite populations to identify other diabetes-associated markers/SNPs and determine their performance in prediction/screening.

- Additional markers/SNPs would need to be considered in certain high-risk populations (e.g., maturity- onset diabetes of the young mutations in patients diagnosed with diabetes and in families segregating for diabetes).

\section{Recommendations of other groups}

- No other organizations have provided recommendations relating specifically to genomic testing/profiling for risk predicting in the general or high-risk populations.

\section{DISCLOSURE}

The authors declare no conflict of interest.

\section{REFERENCES}

1. Centers for Disease Control and Prevention. National Diabetes Fact Sheet: national estimates and general information on diabetes and prediabetes in the United States. Centers for Disease Control and Prevention: Atlanta, GA, 2011

2. Valdez R. Detecting undiagnosed type 2 diabetes: family history as a risk factor and screening tool. J Diabetes Sci Technol 2009;3:722-726.

3. Harrison TA, Hindorff LA, Kim H, et al. Family history of diabetes as a potential public health tool. Am J Prev Med 2003;24:152-159.

4. Köbberling J, Tillil H. Empirical risk figures for first degree relatives of noninsulin dependent diabetics. In: Kobberling J, Tattersall RB (eds). The Genetics of Diabetes Mellitus. Academic Press: London, 1982:201-209.

5. Teutsch SM, Bradley LA, Palomaki GE, et al.; EGAPP Working Group. The Evaluation of Genomic Applications in Practice and Prevention (EGAPP) Initiative: methods of the EGAPP Working Group. Genet Med 2009;11: 3-14.

6. Veenstra DL, Piper M, Haddow JE, et al. Improving the efficiency and relevance of evidence-based recommendations in the era of whole-genome sequencing: an EGAPP methods update. Genet Med 2013;15:14-24.

7. Palomaki GE, Melillo S, Marrone M, Douglas MP. Use of genomic panels to determine risk of developing type 2 diabetes in the general population: a targeted evidence-based review. Genet Med 2013;15:600-611.

8. Knowler WC, Barrett-Connor E, Fowler SE, et al.; Diabetes Prevention Program Research Group. Reduction in the incidence of type 2 diabetes with lifestyle intervention or metformin. N Engl J Med 2002;346:393-403.

9. Florez JC, Jablonski KA, Bayley N, et al.; Diabetes Prevention Program Research Group. TCF7L2 polymorphisms and progression to diabetes in the Diabetes Prevention Program. N Engl J Med 2006;355:241-250.

10. Moore AF, Jablonski KA, McAteer JB, et al.; Diabetes Prevention Program Research Group. Extension of type 2 diabetes genome-wide association scan results in the diabetes prevention program. Diabetes 2008;57:25032510.

11. Florez JC, Jablonski KA, Sun MW, et al.; Diabetes Prevention Program Research Group. Effects of the type 2 diabetes-associated PPARG P12A polymorphism on progression to diabetes and response to troglitazone. J Clin Endocrinol Metab 2007;92:1502-1509.

12. Florez JC, Jablonski KA, Kahn SE, et al. Type 2 diabetes-associated missense polymorphisms KCNJ11 E23K and ABCC8 A1369S influence progression to diabetes and response to interventions in the Diabetes Prevention Program. Diabetes 2007;56:531-536.

13. Jablonski KA, McAteer JB, de Bakker PI, et al.; Diabetes Prevention Program Research Group. Common variants in 40 genes assessed for diabetes incidence and response to metformin and lifestyle intervention in the diabetes prevention program. Diabetes 2010;59:2672-2681.

14. Fisher $E$, Boeing $H$, Fritsche $A$, Doering $F$, Joost HG, Schulze MB. Whole-grain consumption and transcription factor-7-like 2 (TCF7L2) rs7903146: genediet interaction in modulating type 2 diabetes risk. Br J Nutr 2009;101: 478-481.

15. Grau K, Cauchi S, Holst C, et al. TCF7L2 rs7903146-macronutrient interaction in obese individuals' responses to a 10-wk randomized hypoenergetic diet. Am J Clin Nutr 2010;91:472-479.

16. Ioannidis JP, Boffetta P, Little J, et al. Assessment of cumulative evidence on genetic associations: interim guidelines. Int J Epidemio/ 2008;37:120-132.

17. Palomaki GE, Melillo S, Neveux L, et al. Use of genomic profiling to assess risk for cardiovascular disease and identify individualized prevention strategies-a targeted evidence-based review. Genet Med 2010;12:772-784.

18. McBride CM, Bepler G, Lipkus IM, et al. Incorporating genetic susceptibility feedback into a smoking cessation program for African-American smokers with low income. Cancer Epidemiol Biomarkers Prev 2002;11:521-528.

19. Pijl M, Timmermans DR, Claassen $L$, et al. Impact of communicating familial risk of diabetes on illness perceptions and self-reported behavioral outcomes: a randomized controlled trial. Diabetes Care 2009;32: 597-599. 